\title{
Performance Evaluation of the Inveon Dedicated PET Preclinical Tomograph Based on the NEMA NU-4 Standards
}

\author{
Qinan Bao ${ }^{1}$, Danny Newport ${ }^{2}$, Mu Chen ${ }^{3}$, David B. Stout ${ }^{1}$, and Arion F. Chatziioannou ${ }^{1}$ \\ ${ }^{I}$ David Geffen School of Medicine at UCLA, Crump Institute for Molecular Imaging, University of California, Los Angeles, \\ California; ${ }^{2}$ Out of the Box Engineering, Knoxville, Tennessee; and ${ }^{3}$ Siemens Preclinical Solutions, Inc., Knoxville, Tennessee
}

The Inveon dedicated PET (DPET) scanner is the latest generation of preclinical PET systems devoted to high-resolution and high-sensitivity murine model imaging. In this study, we report on its performance based on the National Electrical Manufacturers Association (NEMA) NU-4 standards. Methods: The Inveon DPET consists of 64 lutetium oxyorthosilicate block detectors arranged in 4 contiguous rings, with a 16.1 -cm ring diameter and a 12.7-cm axial length. Each detector block consists of a $20 \times 20$ lutetium oxyorthosilicate crystal array of $1.51 \times 1.51 \times$ $10.0 \mathrm{~mm}$ elements. The scintillation light is transmitted to position-sensitive photomultiplier tubes via optical light guides. Energy resolution, spatial resolution, sensitivity, scatter fraction, and counting-rate performance were evaluated. The NEMA NU4 image-quality phantom and a healthy mouse injected with ${ }^{18} \mathrm{~F}-$ FDG and ${ }^{18} \mathrm{~F}^{-}$were scanned to evaluate the imaging capability of the Inveon DPET. Results: The energy resolution at $511 \mathrm{keV}$ was $14.6 \%$ on average for the entire system. In-plane radial and tangential resolutions reconstructed with Fourier rebinning and filtered backprojection algorithms were below 1.8-mm full width at half maximum (FWHM) at the center of the field of view. The radial and tangential resolution remained under $2.0 \mathrm{~mm}$, and the axial resolution remained under 2.5- $\mathrm{mm}$ FWHM within the central 4-cm diameter of the field of view. The absolute sensitivity of the system was $9.3 \%$ for an energy window of $250-625 \mathrm{keV}$ and a timing window of $3.432 \mathrm{~ns}$. At a $350-$ to $625-\mathrm{keV}$ energy window and a $3.432-n s$ timing window, the peak noise equivalent counting rate was $1,670 \mathrm{kcps}$ at $130 \mathrm{MBq}$ for the mouse-sized phantom and $590 \mathrm{kcps}$ at $110 \mathrm{MBq}$ for the rat-sized phantom. The scatter fractions at the same acquisition settings were $7.8 \%$ and $17.2 \%$ for the mouse- and rat-sized phantoms, respectively. The mouse image-quality phantom results demonstrate that for typical mouse acquisitions, the image quality correlates well with the measured performance parameters in terms of image uniformity, recovery coefficients, attenuation, and scatter corrections. Conclusion: The Inveon system, compared with previous generations of preclinical PET systems from the same manufacturer, shows significantly improved energy resolution, sensitivity, axial coverage, and counting-rate capabilities. The performance of

Received Jul. 26, 2008; revision accepted Nov. 25, 2008.

For correspondence or reprints contact: Arion F. Chatziioannou, 700 Westwood Plaza, Crump Institute for Molecular Imaging, Los Angeles, CA 90095.

E-mail: archatziioann@mednet.ucla.edu

Guest Editor: David Townsend, University of Tennessee Medical Center

COPYRIGHT @ 2009 by the Society of Nuclear Medicine, Inc. the Inveon is suitable for successful murine model imaging experiments.

Key Words: microPET; small-animal PET scanner; performance evaluation; instrumentation; molecular imaging

J Nucl Med 2009; 50:401-408

DOI: 10.2967/jnumed.108.056374

$\mathbf{T}$ omographic systems dedicated to noninvasive, in vivo imaging of preclinical animal models have been widely used at research institutes in recent years $(1,2)$. With the ability to longitudinally image the same subject, each individual animal can serve as its own control. Therefore, intersubject variability can be minimized. Because of the dramatic difference in size between humans and rodents, small-animal PET imposes higher performance requirements than does clinical PET, particularly on image resolution and sensitivity. The resolution and sensitivity improvements are mainly achieved by using smaller crystal sizes, smaller detector ring diameters, and longer axial coverage. With the goal of improving the image quality of laboratory small-animal studies, researchers continually develop new techniques, including new scintillation materials, detectors, electronics, and geometries, to improve the performance of preclinical tomographs.

The Inveon dedicated PET (DPET) is the latest generation of commercial tomographs from Siemens Preclinical Solutions, Inc. DPET incorporates changes in system geometry, detectors, and electronics. This work evaluates the performance of the Inveon system in all aspects, including energy and spatial resolutions, sensitivity, scatter fraction, counting-rate performance, and imaging capabilities, on the basis of the National Electrical Manufacturers Association (NEMA) NU-4 standards for performance evaluation of small-animal PET scanners (3). The NEMA NU-4 standards offer a uniform methodology for smallanimal PET performance evaluation and establish a baseline of system performance in typical imaging conditions. 


\section{MATERIALS AND METHODS}

\section{System Description}

The Inveon DPET is a lutetium oxyorthosilicate (LSO)-based, high-sensitivity, high-resolution preclinical PET scanner used primarily for murine model imaging. The system consists of 64 detector blocks arranged in 4 contiguous rings, with a crystal ring diameter of $16.1 \mathrm{~cm}$ and an axial extent of $12.7 \mathrm{~cm}$. Each detector block is composed of a $20 \times 20$ array of LSO crystals coupled to a positionsensitive photomultiplier tube via a light guide. Each crystal is 10.0 $\mathrm{mm}$ long and has a cross-sectional area of $1.51 \times 1.51 \mathrm{~mm}$. The crystal pitch is $1.59 \mathrm{~mm}$ in both axial and transverse directions.

List-mode data are acquired during measurements. From the listmode data, coincidence events can then be sorted into 3-dimensional (3D) sinograms, with different combinations of span and ring differences, or into 2-dimensional (2D) sinograms by either singleslice rebinning (SSRB) (4) or Fourier rebinning (FORE) (5). Images can be reconstructed using analytic $2 \mathrm{D}$ filtered backprojection (FBP), 3D reprojection (6), or iterative methods, such as orderedsubsets expectation maximization $(7,8)$ and maximum a posteriori (MAP) (9).

The specifications between the Inveon DPET and 3 previous preclinical systems from the same manufacturer are compared in Table 1.

\section{Energy Resolution}

An ${ }^{18} \mathrm{~F}$ point source was placed at the center of the field of view (FOV) to acquire 2D position histograms of each detector in singles mode, with the energy window wide open. A total of 100,000 counts were acquired for each detector pixel. Lookup tables were generated for individual crystal identification (10).

Energy resolution was determined for each crystal in the system and calculated as full width at half maximum (FWHM) of the 511keV energy peak divided by the center of the photopeak value. The mean value of all crystal energy resolutions was calculated, and the maximum and minimum energy resolutions were obtained.

\section{Spatial Resolution}

Spatial resolution was measured with a ${ }^{22} \mathrm{Na}$ point source conforming to the NEMA NU-4 standards. The ${ }^{22} \mathrm{Na}$ point source has a nominal size $(0.3 \mathrm{~mm})$, is embedded in an acrylic cube (10.0-mm extent on all sides), and has a nominal activity $(198,000$ $\mathrm{Bq}$ ). The energy window setting was $350-625 \mathrm{keV}$, and the timing window was $3.432 \mathrm{~ns}$ (the default of the 4 available timing window settings on the Inveon system). ${ }^{22} \mathrm{Na}$ has emission energy $\left(E_{\text {avg }} \beta^{+}=250 \mathrm{keV}\right)$ and positron range $(\sim 0.23 \mathrm{~mm})$ similar to ${ }^{18} \mathrm{~F}$, which is the most widely used positron-emitting isotope. Per the NEMA protocol, the measured spatial resolutions were not corrected for source size, positron range, or photon acolinearity.

The source was fixed in the tomograph and located at 2 axial positions (the center of the axial FOV and one fourth of the axial FOV [31.75 $\mathrm{mm}$ from the center, along the axial direction]). For each of the 2 axial positions, the source was stepped toward the edge of the transverse FOV. For the central $5 \mathrm{~mm}$ of the transverse FOV, the source was stepped at $1-\mathrm{mm}$ increments and then at 5-mm steps up to the edge of the FOV.

The list-mode data acquired at each location were histogrammed into 3D sinograms with delayed events subtracted to correct for random coincidences. Component-based normalization was applied to compensate for the differences in detection efficiency (11). The 3D sinograms were first Fourier rebinned into $2 \mathrm{D}$ sinograms and then reconstructed by $2 \mathrm{D}$ FBP with a ramp filter cut off at the Nyquist frequency, with a zoom selected to achieve a 0.4-mm-pixel in-plane resolution. The axial plane separation was $0.796 \mathrm{~mm}$. The response function was formed by summing 1-dimensional profiles that were parallel to the radial, tangential, and axial directions. A parabolic fit of the peak point and its 2 nearest neighboring points was used to determine the maximum value of the response function. Linear interpolation between adjacent pixels was used to determine the position of half and one tenth of the maximum; the FWHM and full width at tenth maximum were determined for each extracted profile. Volumetric resolution was calculated on the basis of the FWHMs of the radial, tangential, and axial directions.

\section{Sensitivity}

The sensitivity of the system was measured with the same ${ }^{22} \mathrm{Na}$ point source used in the spatial resolution measurement and an ${ }^{18} \mathrm{~F}$

\section{TABLE 1. Comparison of Small-Animal PET Systems}

\begin{tabular}{|c|c|c|c|c|c|}
\hline \multirow[b]{2}{*}{ Category } & \multirow[b]{2}{*}{ Specification } & \multicolumn{4}{|c|}{ System } \\
\hline & & Concorde P4 & Focus120 & Focus220 & Inveon \\
\hline \multirow[t]{4}{*}{ Detector } & Crystal material & LSO & LSO & LSO & LSO \\
\hline & Crystal size (mm) & $2.2 \times 2.2 \times 10$ & $1.51 \times 1.51 \times 10$ & $1.51 \times 1.51 \times 10$ & $1.51 \times 1.51 \times 10$ \\
\hline & Crystal pitch (mm) & 2.45 & 1.59 & 1.59 & 1.59 \\
\hline & Crystal array & $64(8 \times 8)$ & $144(12 \times 12)$ & $144(12 \times 12)$ & $400(20 \times 20)$ \\
\hline \multirow[t]{9}{*}{ System } & No. of detector blocks & 168 & 96 & 168 & 64 \\
\hline & No. of crystals & 10,752 & 13,824 & 24,192 & 25,600 \\
\hline & No. of rings & 32 & 48 & 48 & 80 \\
\hline & No. of crystals per ring & 336 & 288 & 504 & 320 \\
\hline & Ring diameter $(\mathrm{cm})$ & 26.1 & 15 & 25.8 & 16.1 \\
\hline & Gantry aperture (cm) & 22.0 & 12 & 22.0 & 12 \\
\hline & Axial FOV (cm) & 7.8 & 7.6 & 7.6 & 12.7 \\
\hline & Transaxial FOV (cm) & 19.0 & 10.0 & 19.0 & 10.0 \\
\hline & Solid angle $/ 4 \pi$ & 0.29 & 0.45 & 0.28 & 0.62 \\
\hline \multirow[t]{5}{*}{ Dataset } & No. of sinograms & & & & \\
\hline & $3 \mathrm{D}$ & 1,024 & 2,304 & 2,304 & 6,400 \\
\hline & $2 \mathrm{D}$ & 63 & 95 & 95 & 159 \\
\hline & Sinogram size & $192 \times 168$ & $128 \times 144$ & $288 \times 252$ & $128 \times 160$ \\
\hline & Sampling distance $(\mathrm{mm})$ & 1.225 & 0.815 & 0.815 & 0.815 \\
\hline
\end{tabular}


point source. In addition, the sensitivity was also measured with an ${ }^{18} \mathrm{~F}$ line source inserted in a set of concentric aluminum sleeves, which was a more traditional methodology used in the past (12). To reduce the attenuation from the imaging bed, the ${ }^{18} \mathrm{~F}$ and ${ }^{22} \mathrm{Na}$ point sources were taped on a thin piece of cardboard and placed into the scanner FOV. The concentric aluminum tubes were suspended on both ends without other attenuation material in the FOV.

Because of the difficulty of accurately measuring the small nominal activity of a ${ }^{22} \mathrm{Na}$ source in a standard dose calibrator, the ${ }^{22} \mathrm{Na}$ point source was used only to determine the relative sensitivity between different energy and timing window settings. The ${ }^{22} \mathrm{Na}$ source was positioned at the center of the FOV and scanned for 5 min at a fixed timing window of $3.432 \mathrm{~ns}$. Two sets of energy windows were used: one with a fixed lower-level discriminator (LLD) that equaled $350 \mathrm{keV}$ and a changing upper-level discriminator (ULD) from 550 to $700 \mathrm{keV}$ and the other with a fixed ULD that equaled $625 \mathrm{keV}$ and a changing LLD from 250 to $450 \mathrm{keV}$, both at 50-keV steps. The reason for using a solid ${ }^{22} \mathrm{Na}$ source instead of liquid ${ }^{18} \mathrm{~F}$ to measure the relative sensitivity was 2-fold: to simplify the experimental protocol and to avoid the complications of decay correction between different measurements.

The absolute sensitivity was determined by an ${ }^{18} \mathrm{~F}$ point source of approximately $40 \mu \mathrm{L}$ and $42,550 \mathrm{~Bq}$ placed at the tip of a small centrifuge tube and positioned at the center of the scanner FOV. The absolute sensitivity was measured for $5 \mathrm{~min}$ at an energy window of $350-625 \mathrm{keV}$ and a timing window of $3.432 \mathrm{~ns}$. The activity of the ${ }^{18} \mathrm{~F}$ source was measured in a $\gamma$-counter (Wallac 1480 Wizard 3"; PerkinElmer Life Sciences).

The LSO scintillator crystals have intrinsic radioactivity (1315). ${ }^{176} \mathrm{Lu}$ emits $\beta^{-}$particles with an average energy of $420 \mathrm{keV}$, together with $3 \gamma$-photons of 307,202 , and $88 \mathrm{keV}$, respectively (16). The $\beta^{-}$particles and $\gamma$-photons can make a true coincidence if both fall in the preset energy window $(12,17)$.

For each energy window, a background measurement was acquired for $5 \mathrm{~min}$. The histogrammed background true counts were subtracted from the total histogrammed true counts when the ${ }^{22} \mathrm{Na}$ or ${ }^{18} \mathrm{~F}$ point sources were placed inside the scanner. The number of net true coincidences was normalized to the scan duration, divided by the source activity, and corrected for the branching ratio ( 0.906 for ${ }^{22} \mathrm{Na}$ and 0.967 for ${ }^{18} \mathrm{~F}$ ). Attenuations of the $1-\mathrm{cm}^{3}$ cube of ${ }^{22} \mathrm{Na}$ and the centrifuge tube for the ${ }^{18} \mathrm{~F}$ source were not compensated, but a $9 \%$ sensitivity loss was estimated on the basis of the $0.095 \mathrm{~cm}^{-1}$ attenuation of $511-\mathrm{keV}$ photons in water-equivalent material. The absolute sensitivity at the 350 - to $625-\mathrm{keV}$ energy window was determined by the ${ }^{18} \mathrm{~F}$ measurement. On the basis of the relative sensitivity determined by the ${ }^{22} \mathrm{Na}$ source, the absolute sensitivity for the 350 $\mathrm{keV}$ LLD and 625-keV ULD energy window datasets was also calculated.

We used these measurements to investigate the system sensitivity as a function of the energy window. The energy window used in typical studies should be determined on the basis of the tradeoff between absolute sensitivity, scatter fraction, and system background. For our institute, an energy window of 350-625 keV was selected as a compromise for the typical studies we perform.

The sensitivity dependence on the timing window was also measured for 3 min with the same ${ }^{22} \mathrm{Na}$ point source at the 4 available timing window settings $(2.808,3.432,4.056$, and 4.680 $\mathrm{ns}$ ) and a fixed energy window of $350-625 \mathrm{keV}$.
The axial sensitivity profile was measured with a set of concentric aluminum tubes and a plastic tube filled with ${ }^{18} \mathrm{~F}$ solution, with both ends sealed (12). The source tubing was placed inside the smallest metal tube, suspended in the center of the transaxial FOV, and aligned with the axis of the tomograph. The other aluminum tubes were added, one at a time, and the counting rate was measured for each set of metal tubes for $120 \mathrm{~s}$ at the 350 to $625-\mathrm{keV}$ energy window and 3.432-ns timing window. A set of measurements with different thicknesses of aluminum tubes was used to obtain the counting rate without attenuation by exponential fitting. The list-mode data were histogrammed with SSRB without randoms or scatter correction. For each slice of each acquisition and for points farther than $1 \mathrm{~cm}$ from each side of the peak, the values were set to zero. The counts in all lines of response in the sinograms were summed slice by slice, corrected for decay, and scaled by the acquisition time and the $96.7 \%$ positron yield for ${ }^{18} \mathrm{~F}$. The ${ }^{18} \mathrm{~F}$ activity was measured in a Wallac $\gamma$-counter, and the counts in the central $7-\mathrm{cm}$ and the whole axial FOV were added to calculate the sensitivity of mouse- and rat-sized objects, respectively.

\section{Scatter Fraction and Counting-Rate Performance}

Scatter fraction and counting-rate performance were measured using 2 different cylindric polyethylene phantoms that simulated the geometries of a mouse and rat. The design of the phantoms conformed to the NEMA NU-4 standards.

Both phantoms were made of high-density polyethylene $(0.96$ $\left.\mathrm{g} / \mathrm{cm}^{3}\right)$. The mouselike phantom was a 70-mm-long solid cylinder with a $25-\mathrm{mm}$ diameter. A cylindric hole (diameter, $3.2 \mathrm{~mm}$ ) was drilled parallel to the central axis, at a radial distance of $10 \mathrm{~mm}$. The ratlike phantom had similar geometry but larger dimensions (length, $150 \mathrm{~mm}$; diameter, 50-mm). A 3.2-mm-diameter hole was drilled at a radial offset of $17.5 \mathrm{~mm}$.

A ${ }^{11} \mathrm{C}$ solution with a concentration higher than $1,500 \mathrm{MBq} / \mathrm{mL}$ was enclosed in a flexible tube with an outer diameter fitting the 3.2-mm hole. The initial activity in the FOV was measured in a dose calibrator (Atomlab 300; Biodex Medical Systems) and was higher than 500 and $600 \mathrm{MBq}$, respectively, at the start of the acquisition for the mouse- and rat-sized phantoms. The acquisition was performed at the 350- to 625-keV energy and 3.432-ns timing windows. The phantom was centered in the FOV, and data were acquired until the total activity decayed below 10,000 Bq. The random coincidences were measured by the delayed-window technique. The list-mode data were histogrammed into $2 \mathrm{D}$ sinogram sets lasting $5 \mathrm{~min}$ by SSRB, with separate prompts and delays.

For each prompt sinogram (transaxial bin size, $0.815 \mathrm{~mm}$; slice thickness, $0.796 \mathrm{~mm}$ ), all pixels located farther than $8 \mathrm{~mm}$ from the edge of the phantom were set to zero. The profile of each projection angle was shifted so that the peak pixels were aligned with the center pixel of the sinogram. A sum projection was then produced by adding 160 angular projections in each slice and each frame. All pixel counts outside a 14-mm centered band were assumed to be the sum of random, scatter, and intrinsic counts. A linear interpolation between the left and right border of the 14-mm band was used to estimate these nontrue counts under the profile peak. Counts above this line were regarded as true coincidences. Random coincidences were estimated from the delayed sinogram. A background acquisition was obtained at the same energy window and timing window for $16 \mathrm{~h}$ with the cold mouse- and rat-sized phantoms in the FOV. The intrinsic counts were estimated from the background sinogram. 
The scattered counting rate was then calculated by Equation 1:

$$
\mathrm{R}_{\text {scatter }}=\mathrm{R}_{\text {total }}-\mathrm{R}_{\text {true }}-\mathrm{R}_{\text {random }}-\mathrm{R}_{\text {intrinsic }}
$$

where $\mathrm{R}_{\text {scatter }}, \mathrm{R}_{\text {total }}, \mathrm{R}_{\text {true }}, \mathrm{R}_{\text {random, }}$ and $\mathrm{R}_{\text {intrinsic }}$ are the scatter, total, true, random, and intrinsic counting rates, respectively. The scatter fraction (SF) was calculated by Equation 2:

$$
\mathrm{SF}=\frac{\mathrm{R}_{\text {scatter }}}{\mathrm{R}_{\text {scatter }}+\mathrm{R}_{\text {true }}}
$$

The noise equivalent counting rate (NECR) of each of the 5-min frame acquisitions was determined using the following equation $(18,19)$ :

$$
\mathrm{NECR}=\frac{\mathrm{R}_{\text {true }}^{2}}{\mathrm{R}_{\text {total }}+\mathrm{R}_{\text {random }}}
$$

\section{Imaging Studies}

NEMA Phantom Study. The NEMA NU-4 mouse image-quality phantom (3) is composed of the following 3 regions: a main fillable uniform region chamber (diameter, $30 \mathrm{~mm}$; length, 30 $\mathrm{mm}$ ); a lid that attaches to the main fillable region, containing 2 smaller cold region chambers-one that was filled with nonradioactive water and the other with air (both chambers were composed of hollow cylinders-length, $15 \mathrm{~mm}$; outer diameter [OD], $10 \mathrm{~mm}$; and wall thickness, $1 \mathrm{~mm}$ ); and a solid acrylic glass region (diameter, $30 \mathrm{~mm}$; length, $20 \mathrm{~mm}$ ), with 5 fillable rods drilled through (at $7 \mathrm{~mm}$ from the center) with diameters of 1,2,3, 4 , and $5 \mathrm{~mm}$, respectively. The image-quality phantom was filled with ${ }^{18}$ F-FDG solution $(5.1 \mathrm{MBq})$ and acquired at 350 - to $625-$ $\mathrm{keV}$ energy and 3.432-ns timing windows for $20 \mathrm{~min}$. The phantom was placed on a mouse imaging chamber to simulate actual mouse imaging. The activity in the phantom was measured with a Wallac $\gamma$-counter.

A CT transmission scan of the image-quality phantom and the imaging chamber was obtained using a MicroCAT II tomograph (Siemens Preclinical Solutions). The angular sampling was $1^{\circ}$ per projection for a full $360^{\circ}$ scan. The radiographic source was operated at $500 \mu \mathrm{A}$ and $70 \mathrm{kVp}$. Images were reconstructed using a Feldkamp cone-beam algorithm, with a ramp filter cut off at the Nyquist frequency. The reconstructed CT image was registered with the PET emission image to create an attenuation sinogram. The whole 3D PET sinogram data were first Fourier rebinned and then reconstructed by $2 \mathrm{D}$ FBP, with a ramp filter cut off at the Nyquist frequency. Normalization, dead time, random, attenuation (20), and scatter corrections $(21,22)$ were applied.

A 22.5 -mm-diameter and a 10 -mm-high cylindric volume of interest (VOI) was drawn over the center of the uniform region of the image-quality phantom. The average concentration, maximum and minimum values in this VOI, and percentage SD were measured to estimate the noise performance as a measure of uniformity.

The image slices covering the central 10-mm length of the rods were averaged to obtain a single image slice of lower noise. Circular regions of interest (ROIs) were drawn in this image around each rod, with diameters twice the physical diameters of the rods (the exact size of the ROIs is not critical for this measurement). The maximum values in each of these ROIs were measured and were divided by the mean value obtained in the uniformity test to obtain the recovery coefficient (RC) for each rod size.

The transverse image pixel coordinates of the locations with the maximum ROI values were recorded and used to create $10-\mathrm{mm}-$ long line profiles along the rods in the axial direction. The SD of the pixel values measured along each line profile was calculated. The SD of the RC was calculated as follows:

$$
\mathrm{SD}_{\mathrm{RC}}=\mathrm{RC} \sqrt{\left(\frac{\mathrm{SD}_{\text {lineprofile }}}{\mathrm{Mean}_{\text {lineprofile }}}\right)^{2}+\left(\frac{\mathrm{SD}_{\text {background }}}{\text { Mean }_{\text {background }}}\right)^{2}} \cdot \text { Eq. } 4
$$

To test for the accuracy of scatter correction, VOIs were defined in the water- and air-filled cylindric inserts. The diameter of the VOI was $4 \mathrm{~mm}$ and encompassed the central $7.5 \mathrm{~mm}$ in length in the axial direction. The ratio of the mean in each cold region to the mean of the hot uniform area was reported as spillover ratio.

Mouse Study. One healthy mouse was injected with ${ }^{18} \mathrm{~F}-\mathrm{FDG}$ (8.9 MBq), and another was injected with ${ }^{18} \mathrm{~F}^{-}$(9.4 MBq). One and a half hours later, the mice were centered inside the gantry and imaged for $1 \mathrm{~h}$. CT transmission scans of the mice were obtained using a MicroCAT II tomograph with the same protocol as the CT scan for the image-quality phantom. The PET data were reconstructed with FORE +2 D FBP (with a ramp filter cut off at the Nyquist frequency) and MAP reconstructions $(\beta=0.01)$, with all available corrections applied, including attenuation and scatter.

\section{RESULTS}

\section{Energy Resolution}

Energy resolution of the $511-\mathrm{keV}$ photopeak was $14.6 \%$ on average for 25,600 LSO crystals, with $26.9 \%$ and $8.2 \%$, respectively, as the worst and best energy resolutions. The energy resolution of the Inveon DPET was significantly improved, compared with the previously reported $18.5 \%$ (23), $18.3 \%$ (24), and 26\% (25) energy resolutions for the Focus220, Focus120, and microPET P4 (Siemens Preclinical Solutions, Inc.), respectively.

\section{Spatial Resolution}

Figure 1 shows the radial, tangential, and axial components of the FORE- and FBP-reconstructed point source images. At the center of the FOV, the image resolutions in the transverse planes were below 1.8-mm FWHM and remained under 2.0-mm FWHM within the central 4-cmdiameter FOV.

\section{Sensitivity}

Table 2 summarizes the two measurements of the absolute sensitivity, one at a fixed LLD equalling $350 \mathrm{keV}$ and the other at a fixed ULD equalling $625 \mathrm{keV}$.

At the center of the axial and transaxial FOV, the absolute peak sensitivity measured using an energy window of $350-625 \mathrm{keV}$ and a timing window of $3.432 \mathrm{~ns}$ was $6.72 \%$. When the LLD was lowered to $250 \mathrm{keV}$, the absolute sensitivity was $9.32 \%$. This measurement did not include a correction for the self-attenuation of the source. With consideration of self-attenuation, the absolute sensitivity is expected to be higher than $10 \%$ at the 250 - to 625 keV energy window. 

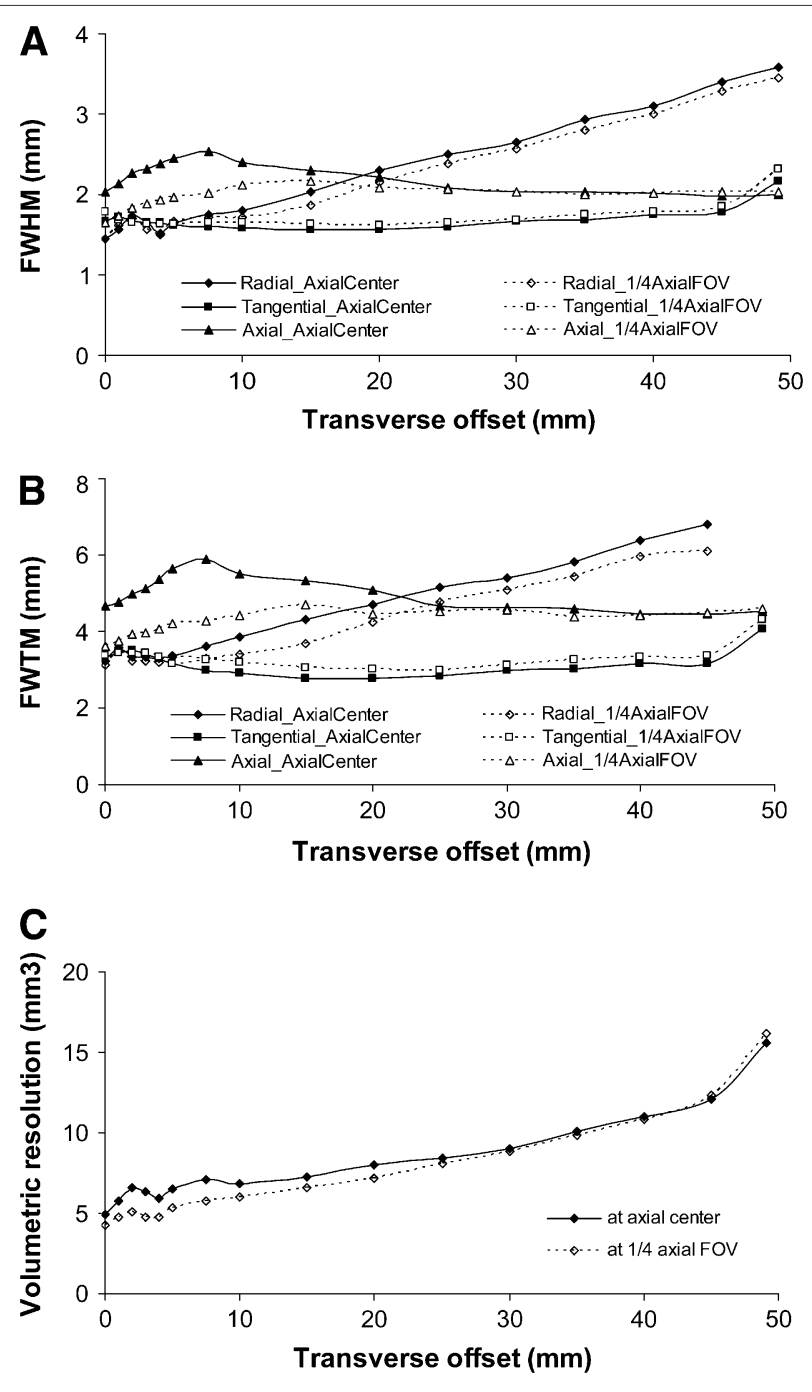

FIGURE 1. FORE +2 D FBP reconstructed image resolution of Inveon DPET system as function of radial offset from center of the FOV. FWHM (A) and FWTM (B) of radial, tangential, and axial image resolutions and volumetric resolutions for point sources located at axial center $(\diamond)$ and $31.75 \mathrm{~mm}$ from axial center $(\diamond)(C)$. FWTM = full width at tenth maximum.

The relative sensitivity measured at different timing windows was compared with the sensitivity measured with a 4.680-ns timing window (the widest timing window available on the Inveon). The measurement shows that the

\begin{tabular}{|c|c|c|c|c|c|}
\hline Absolute sensitivity for. & \multicolumn{5}{|c|}{ Energy window setting (keV) } \\
\hline \multicolumn{6}{|l|}{ Fixed LLD $=350 \mathrm{keV}$} \\
\hline ULD & 550 & 600 & 625 & 650 & 700 \\
\hline Sensitivity (\%) & 6.32 & 6.64 & 6.72 & 6.74 & 6.85 \\
\hline \multicolumn{6}{|l|}{ Fixed ULD $=625 \mathrm{keV}$} \\
\hline LLD & 250 & 300 & 350 & 400 & 450 \\
\hline Sensitivity (\%) & 9.32 & 7.86 & 6.72 & 5.95 & 4.19 \\
\hline
\end{tabular}

sensitivity does not depend significantly on the timing window (within $1.5 \%$ ).

The average sensitivity for a mouse-sized object $(7 \mathrm{~cm})$ and a rat-sized object $(12.7 \mathrm{~cm})$ measured at a 350 - to 625 $\mathrm{keV}$ energy window and a 3.432-ns timing window was $4.0 \%$ and $2.8 \%$, respectively. The axial sensitivity profile is shown in Figure 2.

\section{Scatter Fraction and Counting-Rate Performance}

With an energy window of 350-625 keV and a timing window of $3.432 \mathrm{~ns}$, the peak NECR is 1,670 kcps (achieved at $130 \mathrm{MBq}$ ) for the mouse-sized phantom and $590 \mathrm{kcps}$ (achieved at $110 \mathrm{MBq}$ ) for the rat-sized phantom. The scatter fraction at this acquisition setting is $7.8 \%$ and $17.2 \%$ for the mouse- and rat-sized phantoms, respectively. The NECR as a function of activity is plotted in Figure 3 for the 2 phantoms.

The count loss due to dead time was also investigated on the basis of the mouse-sized phantom data (Fig. 4). The count loss is $25 \%$ at an activity of $50 \mathrm{MBq}$ and $50 \%$ at an activity of $110 \mathrm{MBq}$. The dead-time correction works well up to an activity of $50 \mathrm{MBq}$ (within $1 \%$ accuracy, compared with the expected counts).

\section{Imaging Studies}

NEMA Phantom Study. Figure 5 shows the images of a transverse plane with 5 rods (Fig. 5A), a coronal plane (Fig. 5B), a transverse plane of the uniform region (Fig. 5C), and a profile across the uniform area (Fig. 5D) of the NEMA image-quality phantom.

With FORE + 2D FBP reconstruction, the percentage SD in the uniform region was 5.29 with all corrections applied.

The RCs reconstructed with FBP for 5 different rod sizes from 1- to 5-mm diameter are shown in Figure 6. The RC for the smallest $1-\mathrm{mm}$ rod is 0.17 , and for the largest $5-\mathrm{mm}$ rod the $\mathrm{RC}$ is 0.93 .

The spillover ratios measured in the air- and water-filled chambers of the NEMA phantom after all the corrections had been applied were $-0.57 \%$ and $1.65 \%$, respectively. Without attenuation and scatter correction, the spillover

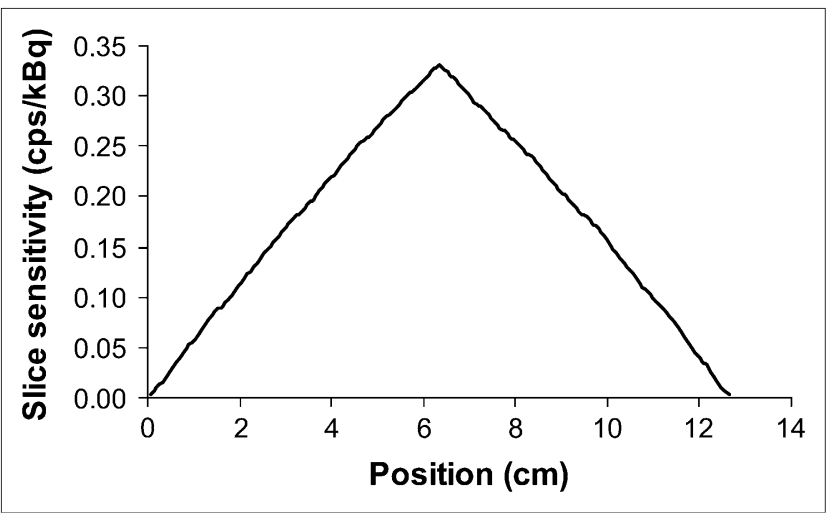

FIGURE 2. Sensitivity profile over axial positions. 


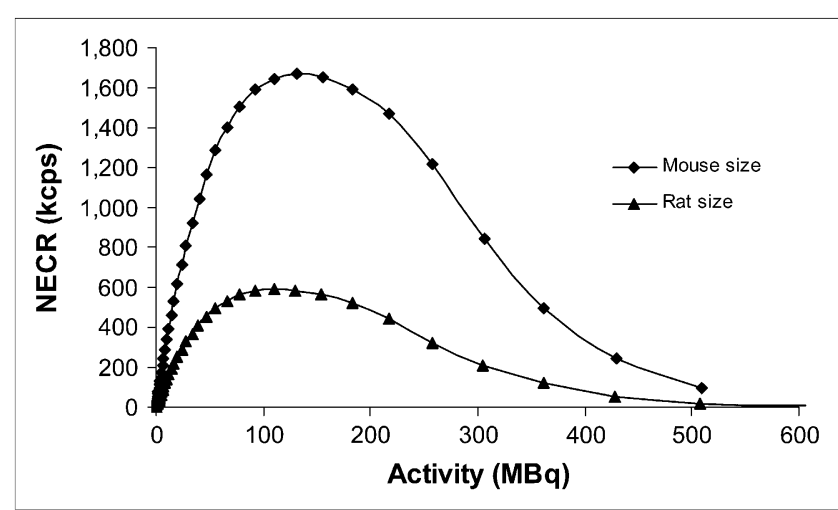

FIGURE 3. NECR as function of total activity for mouseand rat-sized phantoms.

ratios for air- and water-filled chambers were $13.95 \%$ and $-0.21 \%$, respectively. The residual activity is a measurement of correction accuracy. With spillover ratios below $2 \%$ after corrections for both air- and water-filled chambers, the corrections work reasonably well.

Mouse Study. Figure 7 shows the coronal and sagittal images of mice with ${ }^{18} \mathrm{~F}$-FDG and ${ }^{18} \mathrm{~F}^{-}$uptake, respectively. The images were reconstructed with FBP and MAP $(\beta=0.01)$.

\section{DISCUSSION}

The NEMA standards are not meant to produce absolute performance measurements but rather define measurements that can be easily performed, analyzed, and interpreted by the user community. They serve as tools for comparison of imaging instruments under specific operating conditions. These specifications represent a subset of measurements that define the performance of preclinical PET systems for specific imaging tasks.

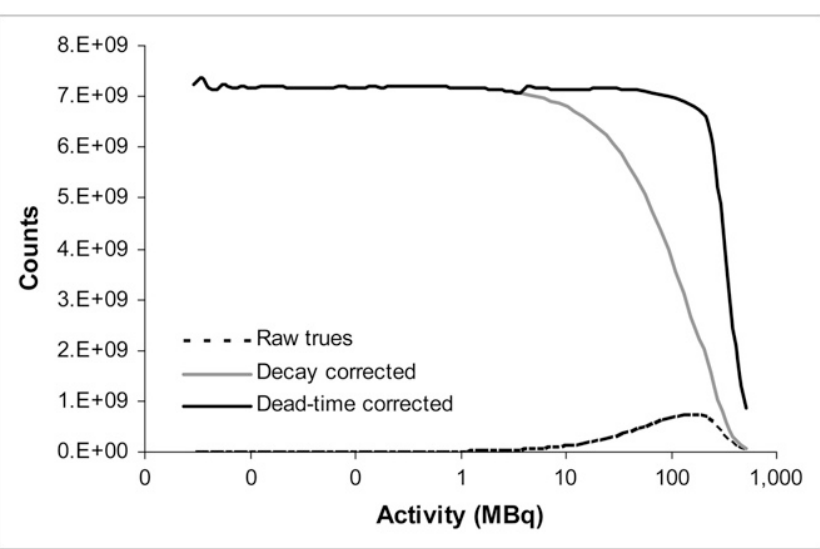

FIGURE 4. Raw counts, decay-corrected counts, and dead time-corrected counts as function of total activity in FOV based on mouse-sized phantom data.

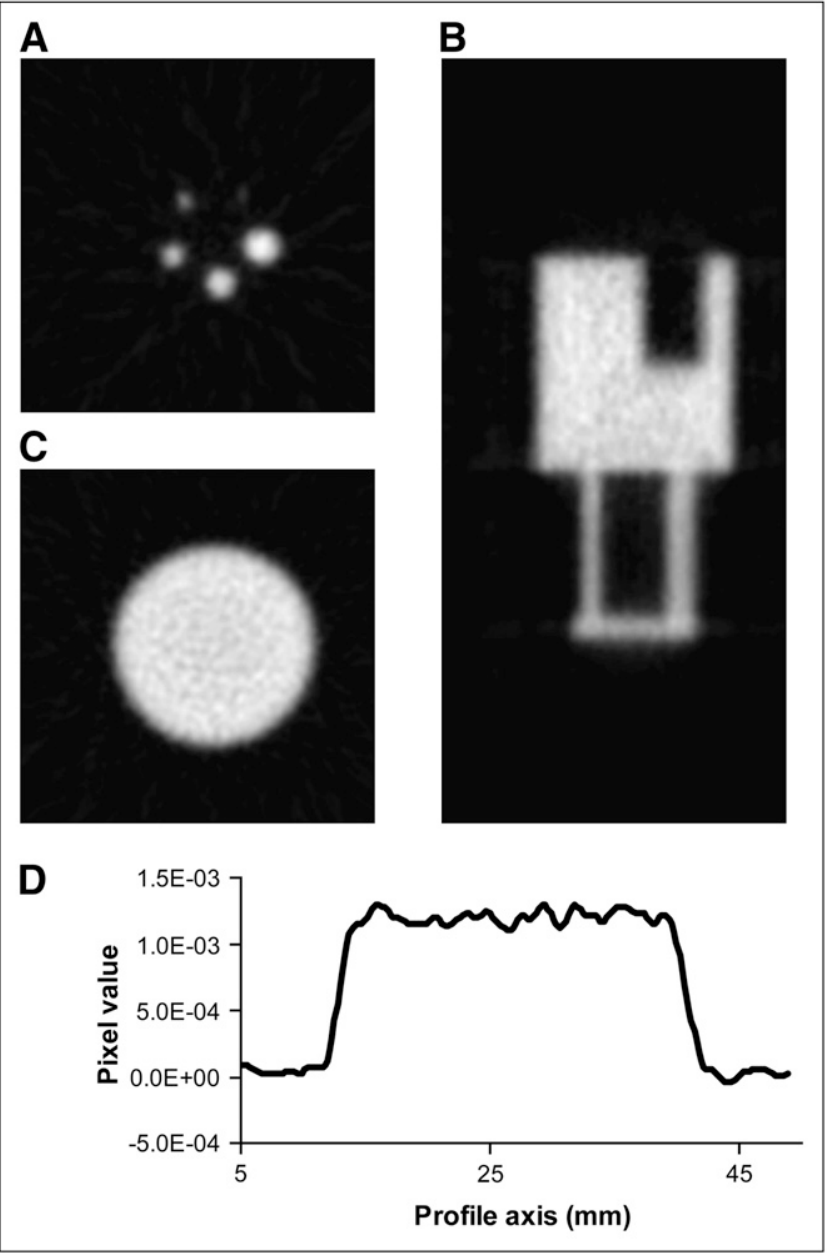

FIGURE 5. Images of NEMA NU-4 image-quality phantom scanned for $20 \mathrm{~min}$ with ${ }^{18} \mathrm{~F}-\mathrm{FDG}(5.1 \mathrm{MBq})$ : transverse plane of 5-rod region (A), coronal view $(B)$, transverse plane of uniform region $(C)$, and profile across uniform area (D).

The energy and spatial resolutions were primarily determined by the scintillator type and individual crystal size of the system. The LSO-based Inveon DPET system has improved energy resolution and spatial resolution similar to the Focus220, which is also based on an LSO scintillator and has the same crystal element size as the Inveon (23). The increase in energy resolution is mainly due to the use of improved light guides, which reduce light loss, in the Inveon. Because of large solid-angle coverage, the sensitivity of the Inveon is significantly higher than that of the previous systems. In addition, because of the increased sensitivity of the Inveon, the peak NECRs for both mouseand rat-sized phantoms are also significantly improved, compared with previous preclinical systems. The scatter fractions cannot be directly compared with previous measurements for other systems $(23,24)$ because a different size of phantom and a different location of the line source insert were used here, per the new NEMA NU-4 standard. In this study, we also have the addition of the NEMA NU-4 image- 


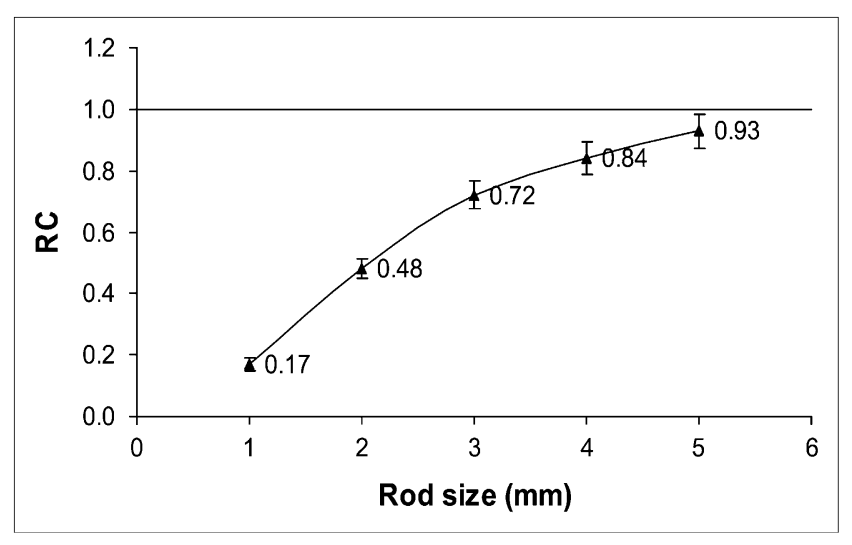

FIGURE 6. RCs for 5 rods of different sizes reconstructed with FBP.

quality phantom that puts together several aspects of the system performance in a single measurement that is relevant to a rather common acquisition protocol.

The measured RCs for different rod sizes are possibly underestimated because of scatter. The RC was calculated by comparing the rod value to the uniform filled background intensity. The rods are surrounded by cold acrylic glass; therefore, there are fewer scattered coincidences in the rod region than in the uniform background area. Although we performed scatter correction during image reconstruction, the scatter correction is possibly less effective for large uniform regions. To investigate this, an increased LLD from 350 to $450 \mathrm{keV}$ was used to image the same image-quality phantom. At that energy window, we saw an increase in the RC values for all rods, demonstrating that the RCs are underestimated at least in part because of scatter. The NEMA mouse image-quality phantom is rather new, and there is limited experience in its use with other systems; therefore, other tomographs need to be investigated to fully evaluate the significance of these findings.

The LSO crystals have an intrinsic background emission. With a wide open energy window, the intrinsic radioactivity will produce a uniform background and affect the ability of detecting low activities in the FOV. The total ${ }^{176} \mathrm{Lu}$ equivalent activity contained in the scintillation crystals is calculated to be $166,500 \mathrm{~Bq}$ based on the percentage of radioactive ${ }^{176} \mathrm{Lu}$. Because of the relative compact geometry and large solid angle of the Inveon DPET, compared with other LSO-based scanners such as the Focus220, the background of the Inveon might be more significant. This LSO background can be reduced by increasing the LLD at the expense of sensitivity. The absolute system sensitivity penalty is about $30 \%$ when the LLD increases from 250 to $350 \mathrm{keV}$.

In the design of the Inveon DPET system, a small detector ring diameter and large axial extent were used to increase the solid-angle coverage and therefore to achieve higher system sensitivity. Because the Inveon DPET has a long axial FOV, it can cover the whole mouse, including the tail, in one scan. The long axial FOV provides the possibility to estimate the total injected activity from the reconstructed image itself. However, because of the relatively small detector ring diameter and long axial extension, compared with other small-animal PET systems, the Inveon is more prone to radial and axial resolution degradation due to the photon penetration at large radial offsets and large ring differences if reconstructed with analytic FBP reconstruction. Statistical reconstruction methods, such as MAP $(9,26)$, should be the preferred image-reconstruction algorithms to obtain highresolution images. Photon penetration and mispositioning of the coincidence events can be modeled with Monte Carlo simulation and incorporated into a system-response matrix to recover the radial and axial resolution losses (27).
A

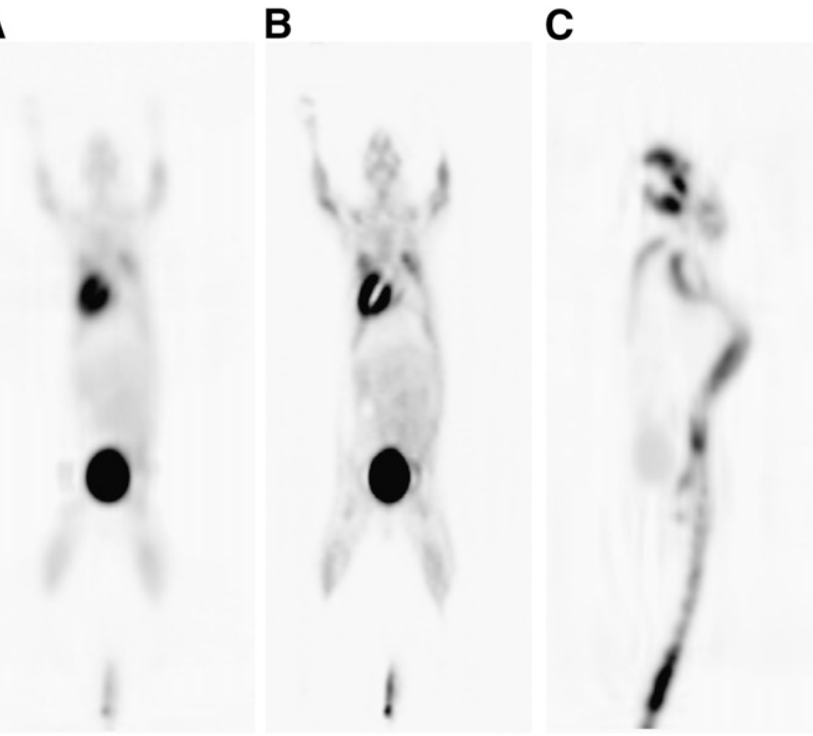

D

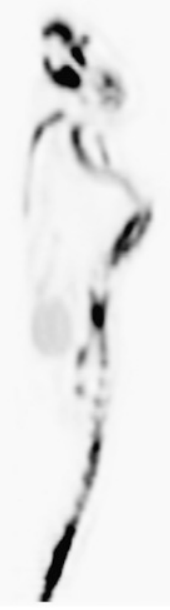

FIGURE 7. Coronal plane of healthy mouse scan with injection of ${ }^{18} \mathrm{~F}-\mathrm{FDG}$ (8.9 $\mathrm{MBq})$ reconstructed with FORE + 2D FBP (A) and MAP (B). Sagittal plane of mouse bone scan with injection of ${ }^{18} \mathrm{~F}^{-}(9.4 \mathrm{MBq})$ reconstructed with FORE + 2D FBP (C) and MAP (D). 


\section{CONCLUSION}

This study evaluated the performance of the small-animal PET Inveon system based on the new NEMA NU-4 protocol. Although direct comparison with previous results acquired with nonstandard sources might not be straightforward, the transverse spatial resolution of the Inveon was similar to that of previous generations of small-animal PET scanners. Absolute sensitivity of the system was $9.3 \%$ for an energy window of 250-625 keV and a timing window of $3.432 \mathrm{~ns}$, which is significantly improved with respect to previous tomographs. The peak NECR of the Inveon system was also greatly increased. The image-quality phantom test demonstrated that the image uniformity and RCs were good, and the scatter correction worked reasonably well. Further improvements in the overall system performance are expected to be realized with fully $3 \mathrm{D}$ iterative reconstruction algorithms that incorporate the estimated system response.

\section{ACKNOWLEDGMENTS}

We thank the NEMA NU-4 Standards Committee and Stephan Siegel, Charles Landen, and Anne Smith from Siemens Preclinical Solutions, Inc., for valuable discussions and technical support. We also thank Sanghee Cho from the Signal and Image Processing Laboratory of the University of Southern California for providing the MAPreconstructed images, Judy Edwards and Waldemar Ladno at the small-animal imaging facility of the Crump Institute for Molecular Irmaging, and the cyclotron team at University of California, Los Angeles. This work was supported by a grant from the National Institutes of Health (SAIRP NIH-NCI 2U24 CA092865).

\section{REFERENCES}

1. Phelps ME. Inaugural article: positron emission tomography provides molecular imaging of biological processes. Proc Natl Acad Sci USA. 2000;97:9226-9233.

2. Cherry SR, Gambhir SS. Use of positron emission tomography in animal research. ILAR J. 2001;42:219-232.

3. National Electrical Manufacturers Association (NEMA). Performance Measurements for Small Animal Positron Emission Tomographs (PETs). NEMA Standards Publication NU 4-2008. Rosslyn, VA: NEMA; 2008.

4. Daube-Witherspoon ME, Muehllehner G. Treatment of axial data in threedimensional PET. J Nucl Med. 1987;28:1717-1724.

5. Defrise M, Kinahan PE, Townsend DW, Michel C, Sibomana M, Newport DF. Exact and approximate rebinning algorithms for 3-D PET data. IEEE Trans Med Imag. 1997;16:145-158.
6. Kinahan PE, Rogers JG. Analytic 3D image reconstruction using all detected events. IEEE Trans Nucl Sci. 1989;36:964-968.

7. Hudson HM, Larkin RS. Accelerated image reconstruction using ordered subsets of projection data. IEEE Trans Med Imag. 1994;13:601-609.

8. Rutao Y, Seidel J, Johnson CA, Daube-Witherspoon ME, Green MV, Carson RE. Performance characteristics of the 3-D OSEM algorithm in the reconstruction of small animal PET images. IEEE Trans Med Imag. 2000;19:798-804.

9. Qi J, Leahy RM, Cherry SR, Chatziioannou A, Farquhar TH. High-resolution 3D Bayesian image reconstruction using the microPET small-animal scanner. Phys Med Biol. 1998;43:1001-1013.

10. Dahlbom M, Hoffman EJ. An evaluation of a two-dimensional array detector for high resolution PET. IEEE Trans Med Imag. 1988;7:264-272.

11. Casey ME, Gadagkar H, Newport D. A component based method for normalization in volume PET. In: Grangeat P, Amans J-L, eds. Third International Conference on Three-Dimensional Image Reconstruction in Radiology and Nuclear Medicine (Computational Imaging and Vision). New York, NY: Kluwer Academic Publishers; 1996:67-71.

12. Laforest R, Longford D, Siegel S, Newport DF, Yap J. Performance evaluation of the microPET-Focus-F120. IEEE Trans Nucl Sci. 2007;54:42-49.

13. Eriksson L, Watson CC, Wienhard K, et al. The ECAT HRRT: an example of NEMA scatter estimation issues for LSO-based PET systems. IEEE Trans Nucl Sci. 2005;52:90-94.

14. Watson CC, Casey ME, Eriksson L, Mulnix T, Adams D, Bendriem B. NEMA NU 2 performance tests for scanners with intrinsic radioactivity. J Nucl Med. 2004; 45:822-826.

15. Yamamoto S, Horii H, Hurutani M, Matsumoto K, Senda M. Investigation of single, random, and true counts from natural radioactivity in LSO-based clinical PET. Ann Nucl Med. 2005;19:109-114.

16. Browne E, Junde H. Nuclear data sheets for $\mathrm{A}=176$. Nucl Data Sheets. 1998;84:337-486.

17. Goertzen AL, Suk JY, Thompson CJ. Imaging of weak-source distributions in LSO-based small-animal PET scanners. J Nucl Med. 2007;48:1692-1698.

18. Strother SC, Casey ME, Hoffman EJ. Measuring PET scanner sensitivity: relating countrates to image signal-to-noise ratios using noise equivalents counts. IEEE Trans Nucl Sci. 1990;37:783-788.

19. Watson CC. Count rate dependence of local signal to noise ratio in positron emission tomography. IEEE Nucl Sci Symp Conf Rec. 2003;4:2817-2821.

20. Chow PL, Rannou FR, Chatziioannou AF. Attenuation correction for small animal PET tomographs. Phys Med Biol. 2005;50:1837-1850.

21. Watson CC, Newport D, Casey ME. A single scatter simulation technique for scatter correction in 3D PET. In: Grangeat P, Amans JL, eds. Fully ThreeDimensional Image Reconstruction in Radiology and Nuclear Medicine. Dordrecht, The Netherlands: Kluwer Academic Publishers; 1996:255268.

22. Watson CC. New, faster, image-based scatter correction for 3D PET. IEEE Trans Nucl Sci. 2000;47:1587-1594.

23. Tai YC, Ruangma A, Rowland D, et al. Performance evaluation of the microPET focus: a third-generation microPET scanner dedicated to animal imaging. J Nucl Med. 2005;46:455-463.

24. Kim JS, Lee JS, Im KC, et al. Performance measurement of the microPET focus 120 scanner. J Nucl Med. 2007;48:1527-1535.

25. Tai C, Chatziioannou A, Siegel S, et al. Performance evaluation of the microPET P4: a PET system dedicated to animal imaging. Phys Med Biol. 2001;46:1845-1862.

26. Chatziioannou A, Qi J, Moore A, et al. Comparison of 3-D maximum a posteriori and filtered backprojection algorithms for high-resolution animal imaging with microPET. IEEE Trans Med Imaging. 2000;19:507-512.

27. Mumcuoglu EU, Leahy RM, Cherry SR, Hoffman E. Accurate geometric and physical response modelling for statistical image reconstruction in high resolution PET. IEEE Nucl Sci Symp Conf Rec. 1996;3:1569-1573. 